\title{
Using UPFC and IPFC Devices Located by a Hybrid Meta-Heuristic Approach to Congestion Relief
}

\author{
Hamid Iranmanesh ${ }^{*}$, Masoud Rashidi-Nejad \\ Islamic Azad University, Jiroft Branch, Jiroft, Iran \\ Email: ${ }^{*}$ iranmanesh_444@yahoo.com
}

Received March 19, 2013; revised April 19, 2013; accepted April 26, 2013

Copyright (C) 2013 Hamid Iranmanesh, Masoud Rashidi-Nejad. This is an open access article distributed under the Creative Commons Attribution License, which permits unrestricted use, distribution, and reproduction in any medium, provided the original work is properly cited.

\begin{abstract}
This paper proposes new methodology for the placement of FACTS devices in transmission systems to reduce congestion. Congestion management comprises congestion relief and congestion cost. The traditional approach to remedying congestion lies in reinforcing the system with additional transmission capacity. Although still feasible, this approach is becoming more and more complex and it is often challenged by the public [1]. Congestion relief can be handled by using FACTS devices, where transmission capability may be improved. Congestion relief using FACTS devices requires a two step approach: first, the optimal location of these devices in the network and then, the settings of their control parameters. UPFC and IPFC have full dynamic control on the transmission parameters, voltage, line impedance and phase angle. Real Genetic Algorithm (RGA) optimization technique is used to solve this congestion relief problem while analytical hierarchy process (AHP) with fuzzy sets is implemented to evaluate RGA fitness function. The results are obtained for modified IEEE 5 bus Test System.
\end{abstract}

Keywords: Congestion Relief; RGA; AHP; Fuzzy Sets; UPFC; IPFC

\section{Introduction}

Electricity industry restructuring and reregulation may dictate maximum power transfer using the existing facilities under transmission open access scheme. Procuring electricity contracts associated with market participants' requirements can cause more challenges considering energy management systems. Reregulation will impose new necessities to power systems such as transmission open access as well as non-discrimination access to the information. Transmission congestion management is an important mechanism in order to solve power transfer bottleneck both in the operation and planning horizons [2]. There are two issues with regards to applying transmission open access that should be considered: the so-called transmission losses and transmission congestion. Congestion is dependent on the network constraints that may show the ultimate transmission capacity, while it can restrict the concurrent electric power contracts [3].

It can be said that, under congestion conditions the price of transferring electricity will be increased. In fact, congestion management is an overall as well as in particular systematic way of improving electricity transfer in

\footnotetext{
${ }^{*}$ Corresponding author.
}

which power systems planning and operating can be regarded.

Transmission congestion is dealing with some restrictions of electricity transfer via transmission network. These restrictions are increased in the presence of open access considering electricity restructuring environment [4]. Under new conditions of power market, more constraints such as economical, environmental problems and transmission rights as financial contracts will be added to technical limitations of transmission capacity [2]. Congestion relief is such a solution to release some blocked capacity of transmission network. In literature, there are some techniques suggested to increase the available transfer capability (ATC). Among the proposed solutions for ATC enhancement, the use of FACTS devices is reported considerably [5]. It can be said that the application of FACTS devices should be based upon the investigation of capital investment as well as operating costs and the impacts of these devices of ATC improvement [6]. On the other hand, the optimum placement of FACTS devices is an important issue in terms of planning horizon [5], especially considering different types of these devices. While from operating point of view, the coordination among these devices is much of interest both by 
researchers and operation engineers.

\section{Transmission Congestion Mathematical Modelling}

In order to study congestion problem, it is needed to define mathematical statements as a proposed model. Mathematical modeling that is implemented in this paper is based upon a multi-objective optimization problem in which some new constraints are added to a conventional optimization model that can be found in literature. In fact, the model includes different terms for objective function such as: improvement of voltage profile, reducing transmission losses and minimizing capital investment for FACTS devices incorporating ATC enhancement. The optimum location as well as the capacity of UPFC can be derived considering the role of these elements.

The study is carried out by implementing a performance index that can be defined as follow:

$$
P I=\sum_{m=1}^{N} \frac{W_{m}}{2 n}\left[\frac{P L_{m}}{P L_{m}^{\max }}\right]^{2 n}
$$

where: $P L_{m}$ is real power transfer in line $m, P L_{m}^{\max }$ is the maximum transfer capacity of line $m, N$ is the number of lines in the network. $W_{m}$ is a non-negative real number to show the importance of $m^{\text {th }}$ transmission line that can be defined as weighting factor and $\mathrm{n}$ is defined as an operating index that is usually less that one. When all transmission lines work at their permissible conditions (non-congestion situation) $P I$ is very low, while if one or more lines are congested it will be increased considerably. To calculate the real power transfer in line $m, \mathrm{DC}$ power flow is applied that is shown in the following relationship:

$$
P l_{m}=\left\{\begin{array}{c}
\sum_{n=1, n \neq s}^{N} S_{m n} P_{n}: m \neq k \\
\sum_{n=1, n \neq s}^{N} S_{m n} P_{n}+P_{j}: m=k
\end{array}\right\}
$$

The coefficients of $S_{m n}$ is the $m n^{\text {th }}$ component of matrix $S$ that is used in DC power flow and $P_{n}$ is the real power injected at bus $\mathrm{n}[7,8]$.

\subsection{UPFC Model}

The UPFC, shown in Figure 1, consists of two switching converters operated from a common DC link. Converter 2 performs the main function of the UPFC by injecting an $\mathrm{AC}$ voltage with controllable magnitude and phase angle in series with the transmission line. The basic function of Converter 1 is to supply or absorb the active power demanded by Converter 2 at the common DC link. This is represented by the current. Converter 1 can also generate or absorb controllable reactive power and pro- vide independent shunt reactive compensation for the line. This is represented by the current. A UPFC can regulate active and reactive power simultaneously. In principle, a UPFC can perform voltage support, power flow control and dynamic stability improvement in one and the same device [9].

\subsection{IPFC Model}

IPFC is mainly used to increase the active power in the line and also to balance the power flow between the lines in the transmission network. In its general form the IPFC employs a number of dc-to-ac converters each providing series compensation for a different line. IPFC is designed as a power flow controller with n number of static synchronous series compensator (SSSC) with a common dc link. For maintaining the power flow stability in the line, power is injected into each bus. The schematic diagram of a simple IPFC with two SSSC is shown in Figure 2 $[10,11]$.

A multi-objective optimization model is represented as a compact form of Equations (3) [7,9].

Subject to the followings:

$\min \frac{P_{i j}}{P_{i j \max }}$ Subject to the followings:

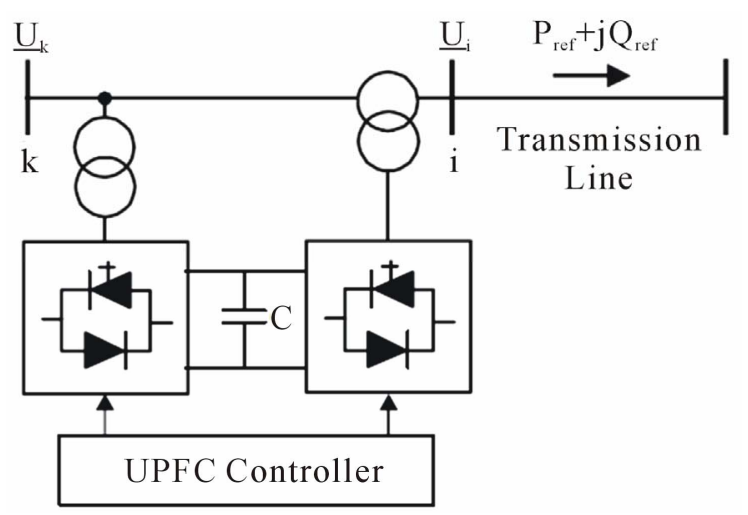

Figure 1. UPFC schematic diagram [9].

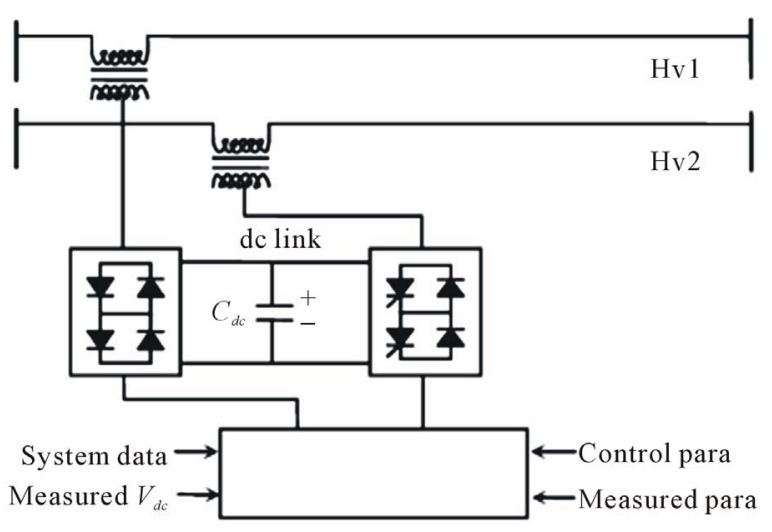

Figure 2. IPFC schematic diagram [10]. 


$$
\begin{aligned}
& P_{g i}-P_{l i}-\sum_{j=1}^{n}\left|V_{i}\right|\left|V_{j}\right|\left(G_{i j-F A C T S} \cos \delta_{i j}+B_{i j-F A C T S} \sin \delta_{i j}\right)=0 \\
& Q_{g i}-Q_{l i}-\sum_{j=1}^{n}\left|V_{i}\right|\left|V_{j}\right|\left(G_{i j-F A C T S} \sin \delta_{i j}-B_{i j-F A C T S} \cos \delta_{i j}\right)=0 \\
& \left|V_{i}\right|_{\min } \leq\left|V_{i}\right| \leq\left|V_{i}\right|_{\max } \\
& P_{i j} \leq 0.8 P_{i j \max } \\
& P_{g i \min } \leq P_{g i} \leq P_{g i \max } \\
& P_{S V C}=0 \\
& Q_{g i \min } \leq Q_{g i} \leq Q_{g i \max } \\
& Q_{s h \text { min }} \leq Q_{s h} \leq Q_{s h \max } \\
& -0.5 X_{m n} \leq X_{s e} \leq 0.6 X_{m n}
\end{aligned}
$$

where: $P_{i j}$ is the real power flow through transmission line $i j ; P_{i j \max }$ is the maximum capacity of line $i j ; P_{l i}$ is the actual real load supply at bus $i ; N$ is bus number of the system; $P_{g i}$ is the real power generation at bus $i$; $Q_{g i}$ is the reactive power generation at bus $i ; Q_{l i}$ is the actual reactive load supply at bus $i ;|V i|$ is the voltage magnitude at bus $i ; G_{i j-F A C T S}, B_{i j-F A C T S}$ are the real/reactive part of the $i j^{\text {th }}$ element of the admittance matrix, which may be a function of the reactance of FACTS Device; $\delta_{i j}$ is the angle difference between the voltage at bus $i$ and that at bus $j ; Q_{g i_{\min }}, Q_{g_{\max }}$ are the minimum/ maximum reactive power generation at generation bus $i$; $|V i|_{\min },|V i|_{\max }$ are the minimum/maximum voltage magnitude at bus I; $X_{s e}$ is the reactance of FACTS Device; $X_{m n}$ is the reactance of the line where FACTS Device has been installed; $P_{s h}$ is the real power generation of FACTS Device; $Q_{s h_{\min }}, Q_{s h_{\max }}$ are the minimum/maximum reactive power generation of FACTS Device [12].

\section{Solution Algorithm}

Heuristic methods may be used to solve complex optimization problems. They are able to give a good solution of a certain problem in a reasonable computation time, but they do not assure to reach the global optimum. GA is a global evolutionary search technique that can result a feasible as well as optimal solutions. Based on the mechanics of natural selection and natural genetics, the GA starts with a population of strings that represent the possible solutions and generates successive populations of strings by combining survival of the fittest among string structures $[13,14]$.

\subsection{Evaluation of Fitness Value via Fuzzy AHP}

The proposed technique for multi-objective goal function will include fuzzy sets theory (FST) [15] which characterized variable $\mathrm{O}$ on $\mathrm{X}$ by its membership as $\mu \mathrm{o}(\mathrm{x}): \mathrm{X} \rightarrow$ $[0,1]$
And analytic hierarchy process (AHP) procedures as following

- FST to conform to mainly qualitative nature of decision factors.

- AHP is for determine importance degree of each alternative.

\subsection{Calculation of Exponential Weighting Values Using AHP}

Analytical hierarchy process (AHP) is a method used to support complex decision-making process by converting qualitative values to numerical values $[16,17]$.

\subsection{Constraints with Unequal Importance}

In case where constraints are of unequal importance it should be ensured that alternatives with higher levels of importance and consequently higher memberships are more likely to be selected. The positive impact of the levels of importance, wi, on fuzzy set memberships is applied through the proposed criterion. It can be realized by associating higher values of wi to constraints. For example, the more important alternative the higher the value associated with it. For example to evaluate fitness function in RGA, it should have higher value for important alternative for this case above process applied as below:

$$
\text { Fitness }=\mu_{c_{1}}^{w_{1}}(x)+\mu_{c_{2}}^{w_{2}}(x)+\cdots+\mu_{c_{N}}^{w_{N}}(x)
$$

\section{Case Study and Results Analysis}

A modified IEEE 5-bus system including 2 generators and 3 loads is selected to implement the proposed methodology for congestion Relief. This system is simulated using Power World software and MATLAB R2010b software where the base MVA and base voltage are assumed to be 100 MVA. In order to enhance power transfer in the congested line, firstly the best location of FACTS devices is derived and control parameters of the allocated devices are then adjusted.

\subsection{Modified IEEE 5-Bus System with Congestion}

As it can be seen in Figure 3, under normal condition, maximum real power is transferred through line 2-1 by amount of $88 \%$ of the permissible line capacity.

Voltage profile of modified IEEE 5-bus system is shown in Figure 4, where at bus 5 the magnitude of bus voltage is $0.747 \mathrm{pu}$. Congestion can be taken into account if real power transfer increases $80 \%$ of the line thermal capacity [18]. By considering congestion condition, it can be said that from congestion point of view there is no security violation while from voltage profile point of view this system needed to be compensated. 


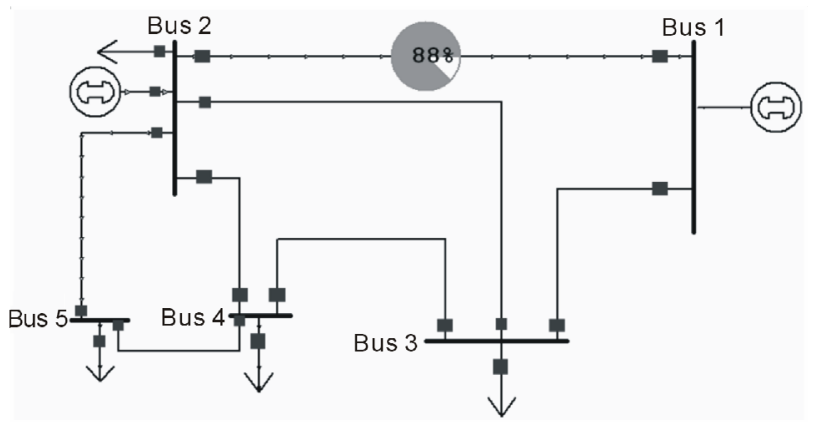

Figure 3. Modified IEEE 5-Bus system with congestion.

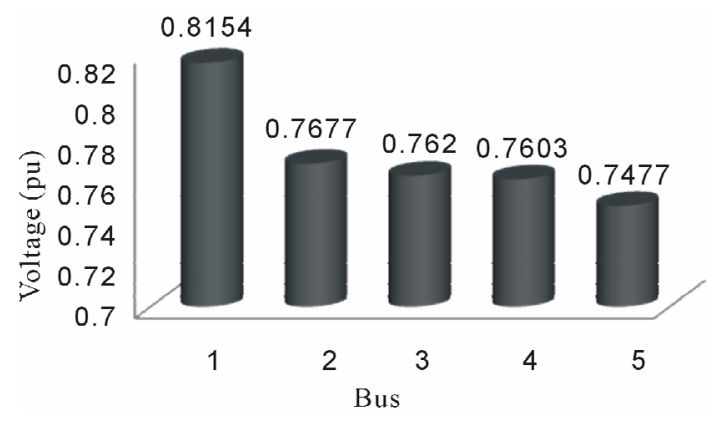

Figure 4. Buses voltage profile.

\subsection{Voltage Profile Improvement and Congestion Relief Using UPFCor IPFC}

In this case goal function includes three objectives: voltage profile, Congestion value and loss. Optimization process tries to relief congestion besides improving voltage profile considering less loss. Multi-objective optimization is handled via fuzzyfying objective function terms. In this respect, membership functions of objective terms are needed to be defined. Typical membership function for voltage of each bus is depicted in Figure 5, while Figure 6 shows congestion membership.

The membership of loss for UPFC or IPFC is assumed as shown in Figure 7.

First of all without any compensation, the electrical system is studied in order to determine the power flow in each of the transmission line and the bus voltages. The power flow results and voltage profile without FACTS Devices are given in Appendix 1 and Figure 4.

In fact, congestion should be relived satisfying minimum loss of UPFC or IPFC while reaching best voltage profile. Moreover, considering AHP criterion, priority factors of objective terms should be derived. It is assumed that congestion is very strong important than voltage profile, while it is absolutely important than the costs of FACTS devices. It can be interpreted that: $\mathrm{P}_{12}=7$, $\mathrm{P}_{21}=\frac{1}{7}, \mathrm{P}_{13}=9, \mathrm{P}_{31}=\frac{1}{9}$

The statement "voltage profile is highly more important than costs of UPFC or IPFC "indicates:

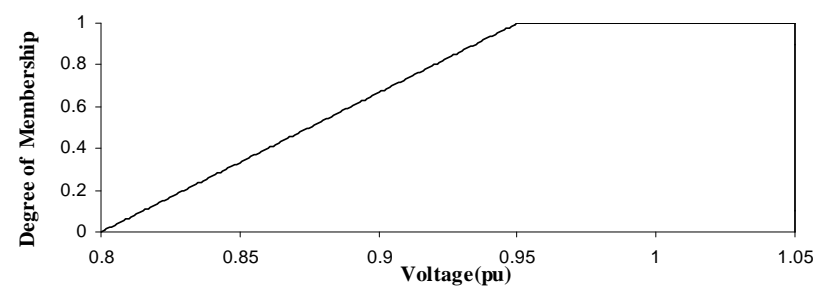

Figure 5. Membership of bus voltages.

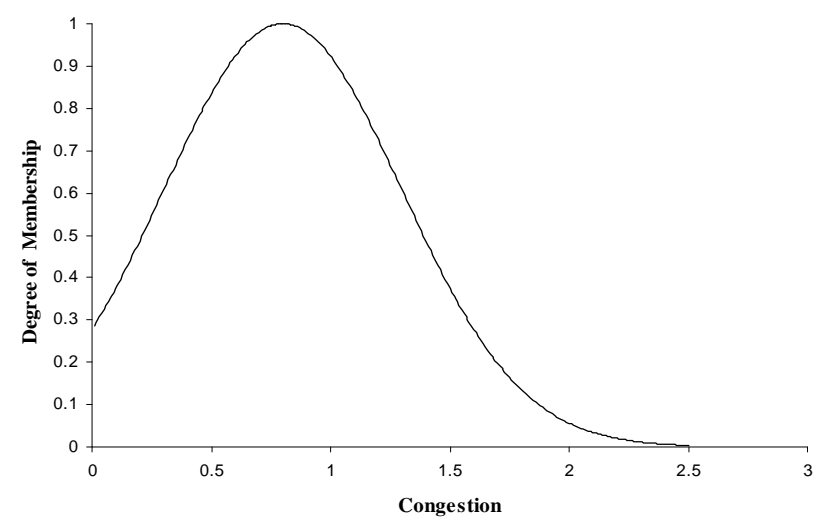

Figure 6. Membership of congestion value.

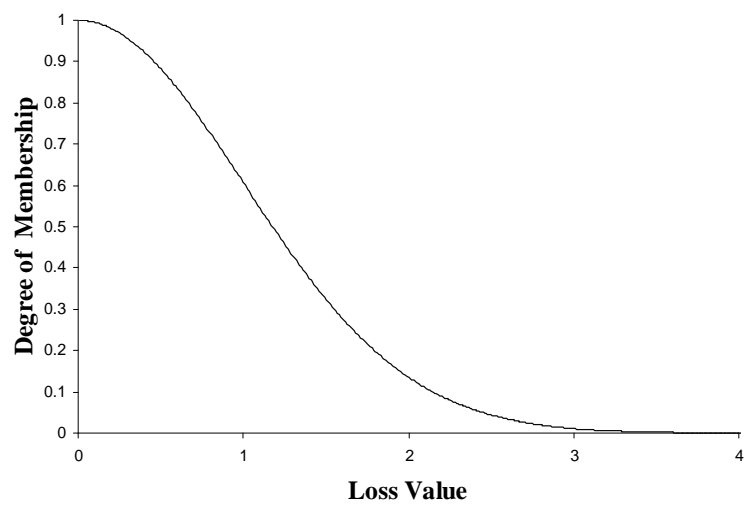

Figure 7. Membership of active power loss.

$$
P_{23}=5, P_{32}=1 / 5
$$

The following weighting $(W)$ vector is obtained via some matrix manipulations.

$$
W=E_{n}=[0.973370 .218670 .068775] T
$$

Fitness value of RGA can be evaluated using Fuzzy-AHP as: Fitness $=\left\{C_{1}^{w_{1}}(x)+C_{2}^{w_{2}}(x)+C_{3}^{w_{3}}(x)\right\}$ where $C_{i}$ is the membership of $i^{\text {th }}$ alternative and $W_{i}$ is exponential weight of $i^{\text {th }}$ alternative.

This procedure can determine the fitness of RGA for each chromosome considering the importance of each constraint as well as objective term. Implementing RGA with the recombination rate of $76 \%$, mutation rate of $3 \%$ and regeneration of $21 \%$ considering ellipsis is applied as it is depicted in Figures 8 and $\mathbf{9}$. These figures show the best solution for UPFC is obtained after 68 generation. It 


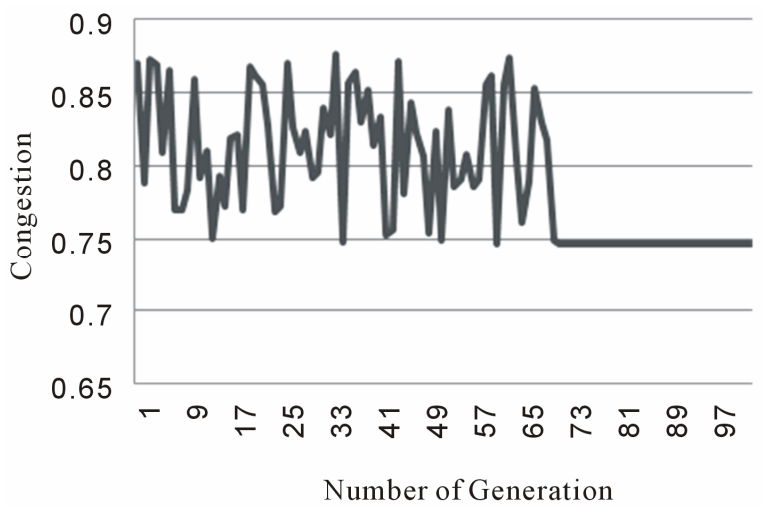

Figure 8. Congestion variations versus number of RGA generation (UPFC).

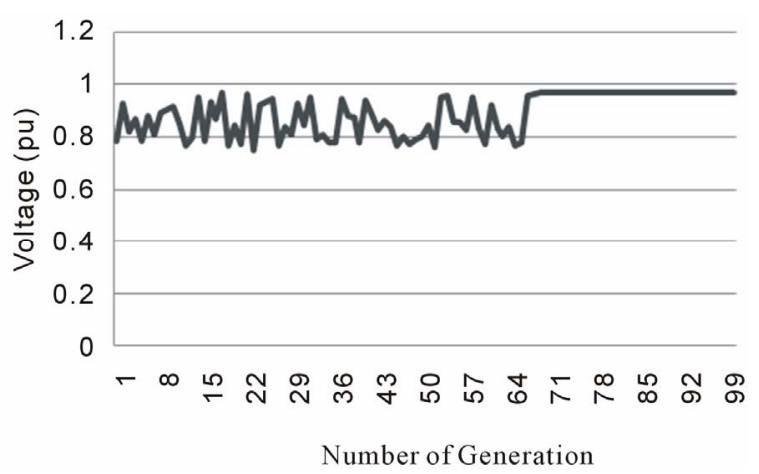

Figure 9. Voltage variations versus number of RGA generation (UPFC).

can be realized that line 3-4 is the candidate for UPFC locations. In this regards the best capacity of those FACTS device is $47.6 \%$ of line reactance which is equal to $0.01428 \mathrm{pu}$ and $15.61 \mathrm{MVAr}$ for respectively.

By installing UPFC at their location, power transfer at line $2-1$ decreases to $74 \%$ of its maximum capacity and the worst voltage is 0.972 which belongs to bus 5 , while it is improved significantly (Appendix 1).

The best solution for IPFC is obtained after 97 generation. But, the two converters of IPFC are embedded in lines between buses 2-4 and 3-4 respectively close to bus 4.

A detailed analysis is carried out to study the effect of IPFC parameters on line flows and bus voltages but, only few results are given for demonstration purpose. The power flow results for IPFC parameters $V_{s e}=0.1 \mathrm{pu}$ and $\theta_{s e}=-150$ are given in Appendix 1.

Figures $\mathbf{1 0}$ and $\mathbf{1 1}$ show the best solution for IPFC is obtained after 97 generation. By installing IPFC at their location, power transfer at line $2-1$ decreases to $73 \%$ of its maximum capacity and the worst voltage is 0.995 which belongs to bus 4 , while it is improved significantly.

Figures 12 and 13 shows bus voltage profile and congestion profile using the allocated UPFC or IPFC.

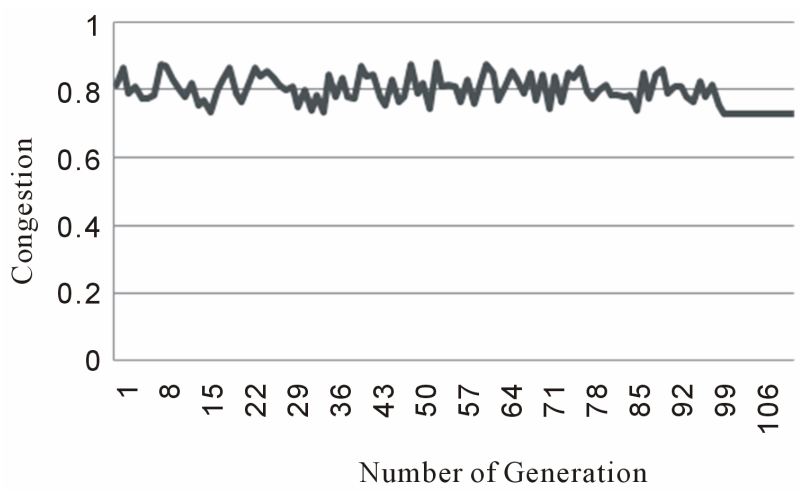

Figure 10. Congestion variations versus number of RGA Generation (IPFC).

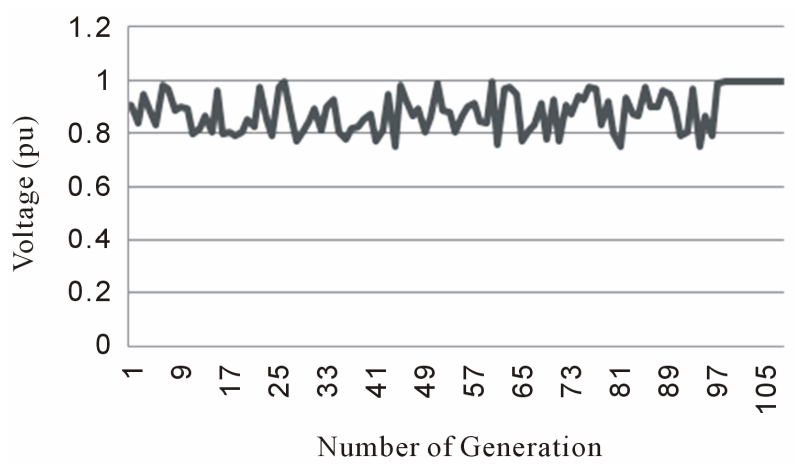

Figure 11. Voltage variations versus number of RGA generation (IPFC).

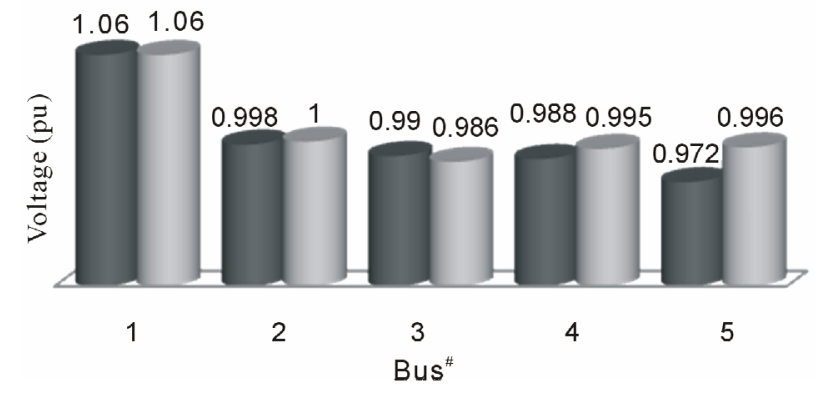

Figure 12. Buses voltage profile using proposed UPFC or IPFC.

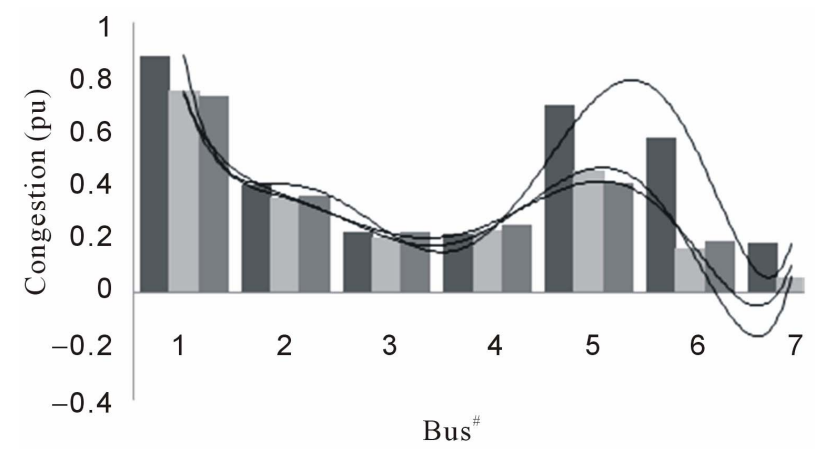

Figure 13. Congestion profile usingn normal condition, proposed UPFC or IPFC. 
The total loss before connecting FACTS Device is $0.066301 \mathrm{MW}$ and after connecting UPFC is reduce to $0.04471 \mathrm{MW}$ and connecting IPFC between two lines the loss is further reduced to $0.02421 \mathrm{MW}$.

\section{Conclusions}

Congestion management is an important issue in the reregulated environment of power systems. Congestion should be relieved in order to use the maximum capacity of transmission networks. It is well known that FACTS technology can control voltage magnitude, phase angle and circuit reactance clearly. Using these devices may redistribute the load flow associated with regulating bus voltages. Therefore, it is worthwhile to investigate the effects of FACTS controllers on the congestion management.

UPFC and IPFC are the main commercially available FACTS controllers. This paper presents an implementation of the RGA associated with Fuzzy-AHP to determine the location and capacity of these devices. The proposed methodology is employed incorporating dimensional serialization valuing mechanism. Case studies and the obtained results show the effectiveness of the suggested criterion significantly.

\section{REFERENCES}

[1] R. Grunbaun, P. Lundberg, G. Stromberg and B. Berggren, "Congestion Relief FACTS: The Key to Congestion Relief," ABB Review, Vol. 2, 2007, pp. 28-32.

[2] Y. H. Song and X. Wang, "Operation of Market-Oriented Power System,” Springer, Berlin, 2003.

[3] K. Singh, N. P. Padhy and J. Sharma, "Influence of Price Responsive Demand Shifting Bidding on Congestion and LMP in Pool-Based Day-Ahead Electricity Markets," IEEE Transactions on Power Systems, Vol. 26, No. 2, 2011, pp. 886-896. doi:10.1109/TPWRS.2010.2070813

[4] M. Esmaili, N. Amjady and H. A. Shayanfar, "MultiObjective Congestion Management by Modified Augmented $\varepsilon$-Constraint Method," Applied Energy, Vol. 88, No. 3, 2011, pp. 755-766. doi:10.1016/j.apenergy.2010.09.014

[5] M. Mandala and C. P. Gupta, "Congestion Management by Optimal Placement of FACTS Device," Power Electronics, Drives and Energy Systems (PEDES) \& 2010 Power India, New Delhi, 20-23 December 2010, pp. 1-7.

[6] M. Joorabian, M. Saniei and H. Sepahvand, "Locating and Parameters Setting of TCSC for Congestion Management in Deregulated Electricity Market," 2011 6th IEEE Conference on Industrial Electronics and Applications (ICIEA), Beijing, 21-23 June 2011, pp. 2185-2190.
[7] K. S. Verma, S. N. Singh, et al., "Location of UPFC for Congestion Management," Electric Power System Research, Vol. 58, No. 2, 2001, pp. 89-96.

[8] N. A. Hosseinipoor and S. M. H. Nabavi, "Social Welfare Maximization by Optimal Locating and Sizing of TCSC for Congestion Management in Deregulated Power Markets," 2010 International Conference on Power System Technology (POWERCON), Hangzhou, 24-28 October 2010, pp. 1-5. doi:10.1109/POWERCON.2010.5666042

[9] C. Bulac, M. Eremaia, R. Balaurescu and V. Stefanescu, "Load Flow Management in the Interconnected Power Systems Using UPFC Devices," 2003 IEEE Bologna Power Tech Conference, Bologna, 23-26 June 2003. doi:10.1109/PTC.2003.1304360

[10] Y. K. Zhang, Y. Zhang and C. Chen, “A Novel Power Injection Model of IPFC for Power Flow Analysis Inclusive of Practical Constraints," IEEE Transactions on Power Systems, Vol. 21, No. 5, 2006, pp. 1550-1556. doi:10.1109/TPWRS.2006.882458

[11] E. Acha, C. R. F. Esquivel, H. A. Pérez and C. A. Camacho, "FACTS: Modelling and Simulation in Power Networks," John Wiley \& Sons Ltd., England, 2004.

[12] A. M. Shan Jiang Gole, U. D. Annakkage and D. A. Jacobson, "Damping Performance Analysis of IPFC and UPFC Controllers Using Validated Small-Signal Models," IEEE Transactions on Power Delivery, Vol. 26, No. 1, 2011, pp. 446-454. doi:10.1109/TPWRD.2010.2060371

[13] D. E. Goldberg, "Genetic Algorithms in Search, Optimization and Machine Learning," Addison-Wesley Longman, Boston, 1989.

[14] S. Sen, S. Chanda, S. Sengupta, A. Chakrabarti and A. De, "Alleviation of Line Congestion Using Multiobjective Particle Swarm Optimization," 2011 International Conference on Electrical Engineering and Informatics (ICEEI), Bandung, 17-19 July 2011, pp. 1-5. doi:10.1109/ICEEI.2011.6021544

[15] L.-X. Wang, "A Course in Fuzzy Systems and Control," Chapter 2, Prentice Hall, Upper Saddle River, 1997.

[16] T. L. Satty, "A Scaling Method for Method for Priorities in Hierarchical Structure," Journal of Math Psychology, Vol. 15, No. 3, 1997, pp. 234-281. doi:10.1016/0022-2496(77)90033-5

[17] W. Ossadnik and O. Lange, "Theory and Methodology, AHP-Based Evaluation of AHP-Software," European Journal of Operational Research, Vol. 118, No. 3, 1999, pp. 578-588. doi:10.1016/S0377-2217(98)00321-X

[18] A. S. Nayak and M. A. Pai, "Congestion Management in Restructured Power Systems Using an Optimal Power Flow Framework," Master Thesis and Project Report, University of Illinois at Urbana-Champaign, PSERC Publication, Tempe, 2002, pp. 2-23. 
Appendix 1

\begin{tabular}{cccccc}
\hline Line & From & To & Congestion without Facts & $\begin{array}{c}\text { Congestion with } \\
\text { UPFC }\end{array}$ & $\begin{array}{c}\text { Congestion with } \\
\text { IPFC }\end{array}$ \\
\hline 1 & 1 & 2 & $88 \%$ & $74 \%$ & $73 \%$ \\
2 & 1 & 3 & $40 \%$ & $35 \%$ & $36 \%$ \\
3 & 2 & 3 & $22 \%$ & $20 \%$ & $22 \%$ \\
4 & 2 & 4 & $22 \%$ & $23 \%$ & $25 \%$ \\
5 & 2 & 5 & $69 \%$ & $45 \%$ & $40 \%$ \\
6 & 3 & 4 & $57 \%$ & $16 \%$ & $18 \%$ \\
7 & 4 & 5 & $18 \%$ & $5 \%$ & $10 \%$ \\
\hline
\end{tabular}

